\title{
ISOMORPHIC SUBGROUPS OF SOLVABLE GROUPS
}

\author{
I. M. ISAACS AND GEOFFREY R. ROBINSON \\ (Communicated by Pham Huu Tiep)
}

\begin{abstract}
Let $H$ and $K$ be isomorphic subgroups of a solvable group $G$, and suppose that $H$ is maximal in $G$. We show that if either $H$ is supersolvable, or a Sylow 2-subgroup of $H$ is abelian, then $K$ is also maximal in $G$.
\end{abstract}

Assume that $H$ and $K$ are isomorphic subgroups of some finite group $G$, and suppose that $H$ is maximal in $G$. In this paper we investigate the question of whether or not $K$ must also be maximal in $G$. In general, the answer is "no" since, for example, we can choose $K$ to be an arbitrary nonabelian simple group and $G=K \times K$. Then $K$ is clearly not maximal in $G$, but the diagonal subgroup $H$ is isomorphic to $K$, and it is not hard to see that $H$ must be maximal in $G$.

The situation is quite different, however, if $G$ is assumed to be solvable. In a posting on the MathOverflow web site, Moshe Newman asked if it can ever happen that a solvable group $G$ has isomorphic subgroups $H$ and $K$, where $H$ is maximal and $K$ is not. Although we have not been able to answer Newman's question fully, we do have some partial results.

Theorem A. Let $H$ be a maximal subgroup of a solvable group $G$, and suppose that $K \subseteq G$ and $K \cong H$. If $H$ has a Sylow tower, and, in particular, if $H$ is supersolvable, then $K$ is maximal in $G$.

Theorem B. Let $H$ be a maximal subgroup of a solvable group $G$, and suppose that $K \subseteq G$ and $K \cong H$. If a Sylow 2-subgroup of $H$ is abelian, then $K$ is maximal in $G$.

Although our proof of Theorem A is relatively elementary, Theorem B seems deeper, and our proof relies on Glauberman's ZJ-Theorem. We begin with some easy general observations.

Lemma 1. Let $G=H U$, where $U \triangleleft G$ and $H \cap U=1$, and suppose that $U$ is solvable. Then $H$ is a maximal subgroup of $G$ if and only if $U$ is a minimal normal subgroup of $G$.

Proof. First suppose that $H$ is maximal, and let $V \triangleleft G$ with $1<V \subseteq U$. To show that $V=U$, note that $V \nsubseteq H$, and thus $H V=G$. We have $V \subseteq U \subseteq H V$, and thus by Dedekind's lemma, $U=(H \cap U) V=V$, as wanted.

Received by the editors February 23, 2014 and, in revised form, March 24, 2014.

2010 Mathematics Subject Classification. Primary 20D10.

Key words and phrases. Solvable group, nilpotent injector, ZJ-Theorem, maximal subgroup. 
Conversely, assume that $U$ is minimal normal in $G$, and note that $U$ is abelian since we are assuming that it is solvable. Let $H \subseteq X<G$. To show that $X=H$, observe that $X=H(X \cap U)$ by Dedekind's lemma, so it suffices to show that $X \cap U=1$. Now $X \cap U<U$ since $H(X \cap U)=X<G=H U$. Also $X U \supseteq H U=G$ and both $X$ and $U$ normalize $X \cap U$, so $X \cap U \triangleleft G$. Since $U$ is minimal normal, it follows that $X \cap U=1$.

Since maximal subgroups of solvable groups have prime-power index, it follows in the situation of Newman's question that $|G: H|$ is a power of some prime $p$. Also $|H|=|K|$, so $|G: H|=|G: K|$, and thus $|G: K|$ is also a power of $p$. It should be clear, therefore, why the following lemma is relevant.

Lemma 2. Let $H \subseteq G$, where $|G: H|$ is a power of a prime $p$. Then $\mathbf{O}_{p}(G) \cap H=$ $\mathbf{O}_{p}(H)$.

Proof. Since $\mathbf{O}_{p}(G) \cap H$ is a normal $p$-subgroup of $H$, we have $\mathbf{O}_{p}(G) \cap H \subseteq \mathbf{O}_{p}(H)$. To prove the reverse containment, consider a Sylow $p$-subgroup $P$ of $G$, and note that $H P=G$. Then $\mathbf{O}_{p}(H)^{G}=\mathbf{O}_{p}(H)^{H P}=\mathbf{O}_{p}(H)^{P} \subseteq P$, and we see that $\mathbf{O}_{p}(H)^{G}$ is a normal $p$-subgroup of $G$. Then $\mathbf{O}_{p}(H) \subseteq \mathbf{O}_{p}(H)^{G} \subseteq \mathbf{O}_{p}(G)$, and we have $\mathbf{O}_{p}(H) \subseteq \mathbf{O}_{p}(G) \cap H$, as required.

We can now begin to address the Newman problem.

Theorem 3. Let $H$ be a maximal subgroup of a solvable group $G$ with index a power of the prime $p$, and suppose that $K \subseteq G$ and $K \cong H$. If $\mathbf{O}_{p}(G) \nsubseteq H$, then $K$ is maximal in $G$.

Proof. We proceed by induction on $|G|$. Let $U=\mathbf{O}_{p}(G)$, and suppose $U \nsubseteq H$, so that $H U>H$, and thus $H U=G$. Write $V=H \cap U$, and observe that $V \triangleleft H$, so $H \subseteq \mathbf{N}_{G}(V)$. Now $V<U$, so $\mathbf{N}_{G}(V) \cap U>V$. But $H \cap U=V$, and we deduce that $\mathbf{N}_{G}(V)>H$. By the maximality of $H$, therefore, $\mathbf{N}_{G}(V)=G$, and we have $V \triangleleft G$. By Lemma 1 applied in the group $G / V$, we conclude that $U / V$ is minimal normal in $G / V$, and in particular, $U / V$ is abelian.

Now write $W=K \cap U$, and observe that $V=\mathbf{O}_{p}(H)$ and $W=\mathbf{O}_{p}(K)$ by Lemma 2, and thus $|V|=|W|$ since $H \cong K$. Since $G=H U$, we have

$$
|G|=\frac{|H||U|}{|V|}=\frac{|K||U|}{|W|}=|K U|,
$$

and we conclude that $K U=G$.

Now $K$ normalizes $W$, and thus $K$ normalizes $W V$. Also, $U$ normalizes $W V$ since $U / V$ is abelian, and thus $W V$ is normal in $K U=G$. Since $U / V$ is a chief factor of $G$, it follows that either $W V=V$ or $W V=U$, and we suppose first that $W V=V$. Then $W \subseteq V$, and since we know that $|V|=|W|$, we conclude that $V=W$. Now $K U=G$ and $K \cap U=V$, and we know that $U / V$ is minimal normal in $G / V$, so it follows by Lemma 1 applied in the group $G / V$ that $K$ is maximal in $G$, as required.

We can now assume that $W V=U$. Write $Y=K \cap H$, and let $X=\theta(Y)$, where $\theta: K \rightarrow H$ is an isomorphism. Then $X$ and $Y$ are isomorphic subgroups of $H$, and we wish to apply the inductive hypothesis in $H$, so we work to show that $X$ is maximal in $H$ and $\mathbf{O}_{p}(H) \nsubseteq X$. 
First, we show that $X$ is maximal in $H$, or, equivalently, that $Y$ is maximal in $K$. We have $G=K U=K W V=K V$, so there exists an isomorphism $\varphi: G / V \rightarrow$ $K /(K \cap V)$, and we see that $\varphi(H / V)=(K \cap H) /(K \cap V)=Y /(K \cap V)$. Since $H / V$ is maximal in $G / V$, it follows that $Y /(K \cap V)$ is maximal in $K /(K \cap V)$, so $Y$ is maximal in $K$, as wanted.

Next, to show that $\mathbf{O}_{p}(H) \nsubseteq X$, it suffices to show that $\mathbf{O}_{p}(K) \nsubseteq Y$. Recall that $W=\mathbf{O}_{p}(K)$, and if $W \subseteq Y$, then $W \subseteq H$, and thus $U=W V \subseteq H$, which is not the case. The inductive hypothesis now implies that $Y$ is maximal in $H$. We have $K H \supseteq K V=G$, so by Dedekind's lemma, intersection with $H$ is an injective map from the set of subgroups $S$ with $K \subseteq S \subseteq G$ into the set of subgroups $T$ with $K \cap H \subseteq T \subseteq H$. Since $K \cap H=Y$ is maximal in $H$, it follows that $K$ is maximal in $G$, as wanted.

Theorem A now follows fairly easily.

Proof of Theorem $A$. We are given a maximal subgroup $H \subseteq G$, where $G$ is solvable, and we assume that $H$ has a Sylow tower, which means that every nontrivial homomorphic image of $H$ has a nontrivial normal Sylow subgroup. Suppose that $K \subseteq G$ and $\theta: H \rightarrow K$ is an isomorphism. We proceed by induction on $|G|$ to show that $K$ is maximal in $G$.

First, suppose that there is a nontrivial subgroup $N \triangleleft G$, where $N \subseteq H$ and $\theta(N)=N$. Then $N \subseteq K$, and $\theta$ induces an isomorphism from $H / N$ to $K / N$. Now $H / N$ and $K / N$ are isomorphic subgroups of $G / N$, and $H / N$ is maximal in $G / N$. Since $H / N$ has a Sylow tower, the inductive hypothesis applies in $G / N$, and we conclude that $K / N$ is maximal in $G / N$, and so $K$ is maximal in $G$.

We can suppose, therefore, that there is no nontrivial subgroup $N$ as in the previous paragraph. If $p$ is the unique prime divisor of $|G: H|$, we can assume by Theorem 3 that $\mathbf{O}_{p}(G) \subseteq H$. By Lemma 2, we see that $\mathbf{O}_{p}(G)=\mathbf{O}_{p}(H)$, and thus $\theta\left(\mathbf{O}_{p}(G)\right)=\theta\left(\mathbf{O}_{p}(H)\right)=\mathbf{O}_{p}(K) \subseteq \mathbf{O}_{p}(G)$, where the containment holds by Lemma 2. It follows that $\theta\left(\mathbf{O}_{p}(G)\right)=\mathbf{O}_{p}(G)$, and since $\mathbf{O}_{p}(G) \triangleleft G$, we have $\mathbf{O}_{p}(G)=1$, and thus also $\mathbf{O}_{p}(H)=1$.

We can certainly assume that $H>1$, and thus by hypothesis, $H$ has a nontrivial normal Sylow $q$-subgroup $Q$ for some prime $q$. Also, $q \neq p$ since $\mathbf{O}_{p}(H)=1$, and thus $Q$ is a full Sylow $q$-subgroup of $G$. If $Q \triangleleft G$, then since $|G: K|$ is a power of $p$, we see that $Q \subseteq K$. Then $Q$ is the unique Sylow $q$-subgroup of $K$, and it follows that $\theta(Q)=Q$. Since $Q$ is nontrivial, this is a contradiction, and we conclude that $Q$ is not normal in $G$.

Now $H \subseteq \mathbf{N}_{G}(Q)<G$, and we deduce that $H=\mathbf{N}_{G}(Q)$. Since $|\theta(Q)|=|Q|$, we see that $\theta(Q)$ is a Sylow $q$-subgroup of $G$, and thus $\theta(Q)=Q^{g}$ for some element $g \in G$. Then $Q^{g}=\theta(Q) \triangleleft \theta(H)=K$, so $K \subseteq \mathbf{N}_{G}\left(Q^{g}\right)=\mathbf{N}_{G}(Q)^{g}=H^{g}$. But $|K|=|H|=\left|H^{g}\right|$, and we deduce that $K=H^{g}$, and thus $K$ is maximal, as required.

In order to prove Theorem B, we need to quote two known results. The first of these is Theorem 4, below, due to H. Lausch [2]. To state it, we say that a nilpotent subgroup $U \subseteq G$ is maximally nilpotent in $G$ if $U$ is not contained in any properly larger nilpotent subgroup of $G$.

Theorem 4 (Lausch). Let $U$ and $V$ be maximally nilpotent subgroups of a finite group $G$, and suppose that $\mathbf{C}_{G}(U \cap V) \subseteq U \cap V$. Then $U$ and $V$ are conjugate in $G$. 
Recall that a nilpotent injector of a solvable group $G$ can be defined to be a maximally nilpotent subgroup of $G$ containing the Fitting subgroup $\mathbf{F}(G)$. Clearly, $G$ has nilpotent injectors, and in fact, $G$ has a unique conjugacy class of such subgroups. (This well-known result is the special case of the following corollary obtained by taking $H=G$.)

Corollary 5. Let $H \subseteq G$, where $G$ is solvable, and suppose that $|G: H|$ and $|\mathbf{F}(G)|$ are coprime. Let $U$ and $V$ be nilpotent injectors of $H$ and $G$, respectively. Then $U$ and $V$ are conjugate in $G$.

Proof. Let $F=\mathbf{F}(G)$, and write $\pi$ to denote the set of prime divisors of $|F|$. Since $F \subseteq V$ and $V$ is nilpotent, the Hall $\pi^{\prime}$-subgroup $Q$ of $V$ satisfies $Q \subseteq \mathbf{C}_{G}(F) \subseteq F$, and thus $Q=1$, and we see that $V$ is a $\pi$-group. Now $|G: H|$ is a $\pi^{\prime}$-number, so $H$ contains a Hall $\pi$-subgroup of $G$, and thus $H$ contains some conjugate $W$ of $V$ in $G$. It suffices to show that $U$ and $W$ are conjugate.

Since $V$ is maximally nilpotent in $G$ and contains $F$, we see that $W$ is also maximally nilpotent in $G$, and it too contains $F$. Then $F \subseteq H$, and hence $F \subseteq$ $\mathbf{F}(H) \subseteq U$. Now $U$ and $W$ are maximally nilpotent in $H$ and $F \subseteq U \cap W$. We have

$$
\mathbf{C}_{H}(U \cap W) \subseteq \mathbf{C}_{G}(F) \subseteq F \subseteq U \cap W,
$$

and thus $U$ and $W$ are conjugate in $H$ by Theorem 4 .

We also need the following version of the Glauberman ZJ-Theorem [1]. To state it, we recall that for a $p$-group $U$, there are several different definitions in the literature of a characteristic subgroup denoted $\mathbf{J}(U)$, and unfortunately, these definitions often yield different subgroups. For our purposes, we define $\mathbf{J}(U)$ to be the subgroup generated by all abelian subgroups of $U$ having largest possible order, and we do not require that $U$ be a $p$-group.

Theorem 6 (Glauberman). Let $P \in \operatorname{Syl}_{p}(G)$, where $p>2$, and assume that a Sylow 2-subgroup of $G$ is abelian. Suppose that $\mathbf{O}_{p}(G) \supseteq \mathbf{C}_{G}\left(\mathbf{O}_{p}(G)\right)$. Then $\mathbf{Z}(\mathbf{J}(P))$ is characteristic in $G$.

Our application of Theorem 6 will be to solvable groups, so by the Hall-Higman lemma, the condition that $\mathbf{O}_{p}(G) \supseteq \mathbf{C}_{G}\left(\mathbf{O}_{p}(G)\right)$ will automatically be satisfied if $\mathbf{O}_{p^{\prime}}(G)=1$.

Theorem 7. Let I be a nilpotent injector of a solvable group $G$, and suppose that a Sylow 2-subgroup of $G$ is abelian. Then $\mathbf{Z}(\mathbf{J}(I))$ is characteristic in $G$.

Proof. For each prime $p$, we show that $\mathbf{Z}(\mathbf{J}(P)) \triangleleft G$, where $P$ is the Sylow $p$ subgroup of $I$. To see this, decompose $\mathbf{F}(G)=A \times B$, where $A$ is a $p$-group and $B$ is a $p^{\prime}$-group. Then $A \subseteq \mathbf{F}(G) \subseteq I$, and so $A \subseteq P$. Also, since $I$ is nilpotent and $P$ and $B$ are subgroups with coprime orders, we have $P \subseteq \mathbf{C}_{G}(B)$.

If $p=2$, then $P$ is abelian, and thus $P$ centralizes $A$ as well as $B$, and we have $P \subseteq \mathbf{C}_{G}(\mathbf{F}(G)) \subseteq \mathbf{F}(G)$. Then $P=A$, so $\mathbf{Z}(\mathbf{J}(P))=A \triangleleft G$, as wanted. We can thus assume that $p \neq 2$.

Write $C=\mathbf{C}_{G}(B)$, and recall that $P \subseteq C$. We argue that, in fact, $P$ is a full Sylow $p$-subgroup of $C$. To see this, let $P \subseteq S \in \operatorname{Syl}_{p}(C)$, and note that 
since $S \subseteq C$, the $p$-group $S$ centralizes the nilpotent $p^{\prime}$-group $B$. Then $S B$ is nilpotent, and so $S B$ is contained in some maximally nilpotent subgroup $U$ of $G$. Now $A \subseteq P \subseteq S$, so $\mathbf{F}(G)=A B \subseteq S B \subseteq U$, and thus $U$ is a nilpotent injector of $G$. Then $U$ is conjugate to $I$, so $S$ is a $p$-subgroup of a conjugate of $I$. Since $P$ is a Sylow $p$-subgroup of $I$, it follows that $|S| \leq|P|$, and hence $S=P$ and $P \in \operatorname{Syl}_{p}(C)$, as claimed.

Let $M=\mathbf{O}_{p^{\prime}}(C)$, so $M$ centralizes $B$ since $C=\mathbf{C}_{G}(B)$. Also, $A$ is a normal p-subgroup of $C$, so $M$ centralizes $A$, and thus $M \subseteq \mathbf{C}_{G}(\mathbf{F}(G)) \subseteq \mathbf{F}(G)$. It follows that $M \subseteq B$, and hence $M \subseteq \mathbf{Z}(C)$.

Write $Z=\mathbf{Z}(\mathbf{J}(P))$, and recall that our goal is to show that $Z \triangleleft G$. The image of $Z$ under the natural isomorphism from $P$ to $P M / M$ is $Z M / M$, and it follows that $Z M / M=\mathbf{Z}(\mathbf{J}(P M / M))$. Since $P M / M$ is a Sylow $p$-subgroup of $C / M$ and $\mathbf{O}_{p^{\prime}}(C / M)$ is trivial, we can apply Theorem 6 to deduce that $Z M / M$ is characteristic in $C / M$. Also, $C \triangleleft G$, so $C / M \triangleleft G / M$, and thus $Z M / M \triangleleft G / M$, and we have $Z M \triangleleft G$. But $Z$ is a normal Sylow $p$-subgroup of $Z M$ since $M$ is central in $C$, and thus $Z \triangleleft G$, as wanted.

Now $I$ is nilpotent, so $\mathbf{J}(I)$ is the product of the subgroups $\mathbf{J}(P)$, as $P$ runs over the Sylow subgroups of $I$. Then $\mathbf{Z}(\mathbf{J}(I))$ is the product of the subgroups $\mathbf{Z}(\mathbf{J}(P))$, and since each of these factors is normal in $G$, we see that $\mathbf{Z}(\mathbf{J}(I)) \triangleleft G$. Since the nilpotent injectors of $G$ are conjugate, it follows that the subgroup $\mathbf{Z}(\mathbf{J}(I))$ is independent of the choice of the particular nilpotent injector $I$, and hence $\mathbf{Z}(\mathbf{J}(I))$ is characteristic in $G$.

We can now prove Theorem B.

Proof of Theorem $B$. We are given a maximal subgroup $H \subseteq G$, where $G$ is solvable, and we assume that a Sylow 2-subgroup of $H$ is abelian. Suppose that $K \subseteq G$ and $\theta: H \rightarrow K$ is an isomorphism. We proceed by induction on $|G|$ to show that $K$ is maximal in $G$.

Using exactly the same reasoning as in the proof of Theorem A, we can assume that there exists no nontrivial subgroup $N \triangleleft G$ such that $N \subseteq H$ and $\theta(N)=N$. Also, as in the proof of Theorem A, we can assume that $\mathbf{O}_{p}(G)=1$, where $p$ is the unique prime divisor of $|G: H|=|G: K|$. Then $\mathbf{F}(G)$ is a $p^{\prime}$-group, and it follows by Corollary 5 that the nilpotent injectors of $H$ and of $K$ are nilpotent injectors of $G$.

Now let $U$ be a nilpotent injector of $H$, and write $V=\theta(U)$ so that $V$ is a nilpotent injector of $K$. Then $U$ and $V$ are nilpotent injectors of $G$, and hence they are conjugate in $G$, and we can write $V=U^{g}$ for some element $g \in G$. We now have

$$
\theta(\mathbf{Z}(\mathbf{J}(U)))=\mathbf{Z}(\mathbf{J}(\theta(U)))=\mathbf{Z}(\mathbf{J}(V))=\mathbf{Z}\left(\mathbf{J}\left(U^{g}\right)\right)=\mathbf{Z}(\mathbf{J}(U))^{g},
$$

so if $\mathbf{Z}(\mathbf{J}(U)) \triangleleft G$, then $\theta(\mathbf{Z}(\mathbf{J}(U)))=\mathbf{Z}(\mathbf{J}(U))$, and this is a contradiction since $\mathbf{Z}(\mathbf{J}(U))$ is nontrivial. By Theorem 7 , however, we have $\mathbf{Z}(\mathbf{J}(U)) \triangleleft H$, and thus $H=\mathbf{N}_{G}(\mathbf{Z}(\mathbf{J}(U)))$ by the maximality of $H$. Then

$$
H^{g}=\mathbf{N}_{G}\left(\mathbf{Z}(\mathbf{J}(U))^{g}\right)=\mathbf{N}_{G}(\mathbf{Z}(\mathbf{J}(V))) \supseteq K,
$$

where the final containment follows by Theorem 7. Since $\left|H^{g}\right|=|H|=|K|$, we have $H^{g}=K$, and thus $K$ is maximal in $G$. 


\section{REFERENCES}

[1] George Glauberman, A characteristic subgroup of a p-stable group, Canad. J. Math. 20 (1968), 1101-1135. MR0230807 (37 \#6365)

[2] Hans Lausch, Conjugacy classes of maximal nilpotent subgroups, Israel J. Math. 47 (1984), no. 1, 29-31, DOI 10.1007/BF02760560. MR736062 (85k:20058)

Department of Mathematics, University of Wisconsin, 480 Lincoln Drive, Madison, WISCONSIN 53706

E-mail address: isaacs@math.wisc.edu

Heilbronn Institute for Mathematical Research, University of Bristol, Bristol BS8 1TW, United Kingdom - And - Institute of Mathematics. University of Aberdeen, Aberdeen, AB24 3UE, Scotland

E-mail address: g.r.robinson@abdn.ac.uk 